\title{
Doença de Forestier: uma causa de disfagia a ser lembrada
}

\author{
Forestier's disease: a cause of dysphagia to recall
}

\author{
Francisco Otavio Camargo Pereira ${ }^{1}$, Flavio Ramalho Romero ${ }^{1}$, Kleber Carlos Azevedo Junior ${ }^{1}$, \\ Ismael Augusto Silva Lombardi ${ }^{1}$, Priscila Watson Ribeiro ${ }^{1}$, Roberto Colichio Gabarra ${ }^{1}$, Marco Antonio Zanini ${ }^{1}$
}

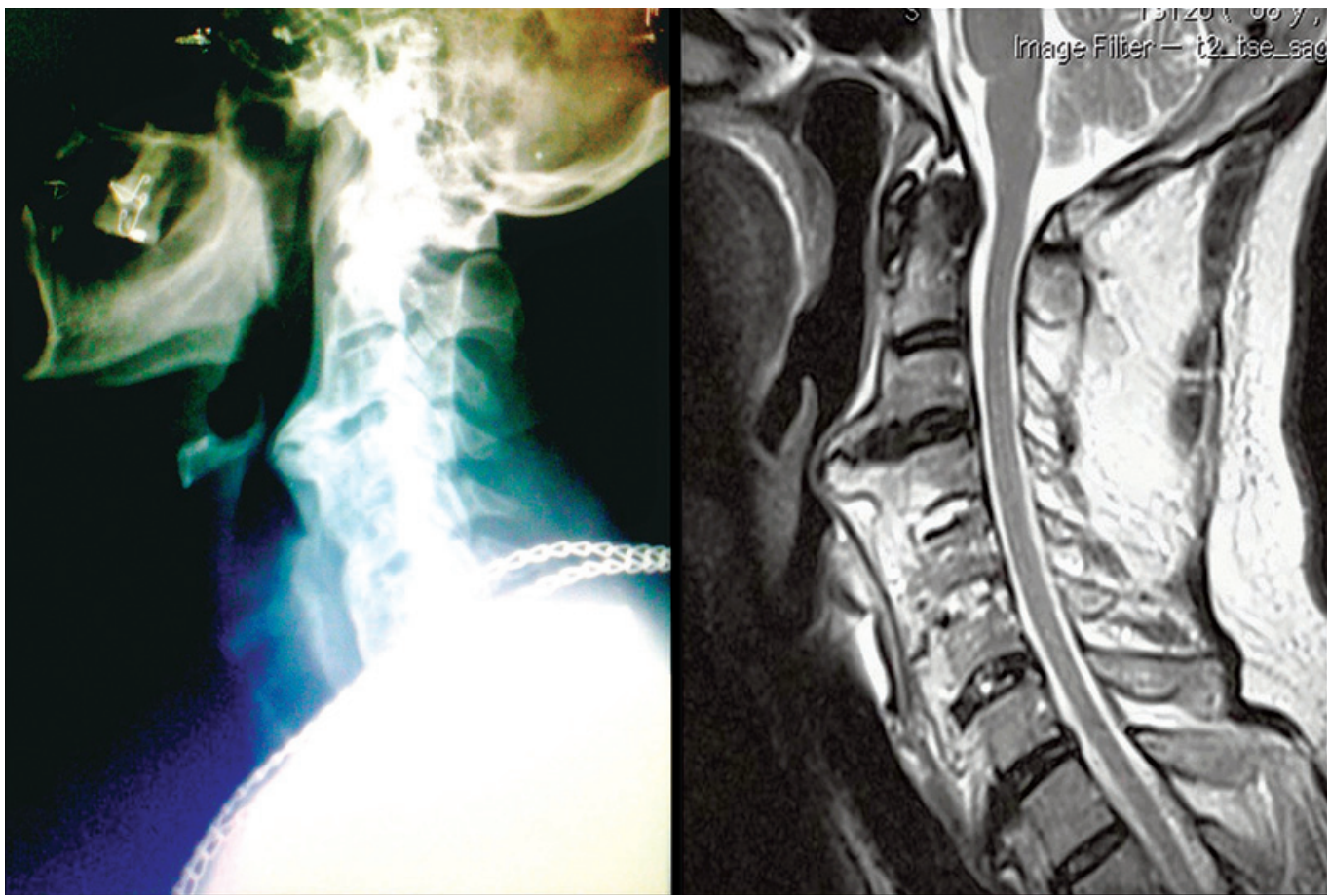

Raio $\mathrm{X}$ cervical em perfil e ressonância magnética sagital ponderada em T2. Observamos extensa calcificação em frente aos corpos vertebrais de C2 a C6 na topografia do ligamento longitudinal anterior. 0 esôfago e a via aérea apresentam-se comprimidos posteriormente, determinando um segmento estenosado, o que dificultou a intubação orotraqueal na ocasião do procedimento cirúrgico

Paciente do gênero masculino, 68 anos, portador de doença de Chagas, hipertensão arterial e diabetes mellitus tipo 2. Acompanhado em hospital quaternário pelas especialidades Otorrinolaringologia e Gastroenterologia devido à quadro crônico de disfagia e odinofagia para alimentos sólidos, com a hipótese diagnóstica não confirmada de megaesôfago chagásico.

Durante investigação complementar foi submetido à radiografia cervical simples em perfil, a qual evidenciou compressão extrínseca esôfago-laríngea secundária à osteófito exuberante, no nível C3-C4. Na radiografia, puderam ser observados ainda acometimento dos níveis adjacentes (C2-C3, C4-C5 e C5-C6), ossificação do ligamento longitudinal anterior e preservação da altura dos espaços discais. Tais achados, somados à ausência de comprometimento radiológico das articulações sacroilíacas, correspondem aos critérios de Resnick ${ }^{(1)}$ encontrados na hiperostose esquelética idiopática difusa (DISH, sigla do inglês diffuse idiopathic skeletal hyperostosis), descrita primeiramente em 1950 por J. Forestier. ${ }^{(2)}$

\footnotetext{
Universidade Estadual Paulista "Júlio de Mesquita Filho", Botucatu, SP, Brasil.

Autor correspondente: Francisco Otavio Camargo Pereira - Distrito de Rubião Junior, s/n - CEP: 18618-970 - Botucatu, SP, Brasil - Tel.: (14) 3880-1220 - E-mail: franotavio@hotmail.com Data de submissão: 20/10/2012 - Data de aceite: 2/12/2013
}

DOI: 10.1590/S1679-45082014AI2659 
A doença de Forestier caracteriza-se por proliferação óssea em locais de inserção dos ligamentos e tendões $^{(3,4)}$ (entesopatia), sendo que a maior parte dos pacientes é assintomática e a doença é descoberta de forma incidental ou em investigação de outros sintomas. Acomete com maior frequência indivíduos do gênero masculino acima dos 50 anos, sendo observada uma correlação com o diabetes. ${ }^{(5)} \mathrm{O}$ paciente em questão apresentava hiperglicemia de difícil controle, a despeito de tratamento clínico otimizado.

Os principais diagnósticos diferenciais ${ }^{(6)}$ são a espondilite anquilosante, que acomete indivíduos mais jovens e é mais sintomática, e a espondilodiscartrose, na qual ocorrem osteófitos de tração e não há comprometimento do ligamento longitudinal anterior.

De modo geral a DISH não apresenta tratamento específico, uma vez que a evolução é lenta, e os pacientes são em sua maioria assintomáticos. ${ }^{(7)} \mathrm{O}$ tratamento clínico medicamentoso, composto por mudança de hábitos alimentares e pelo uso de relaxantes musculares associado à fisioterapia, é uma boa opção para os casos de sintomatologia leve a moderada. Em nosso caso, optamos pelo tratamento cirúrgico devido à sintomatologia grave e ao comprometimento da qualidade de vida do paciente. A osteofitectomia cirúrgica por acesso cervical anterolateral foi realizada sem intercorrências, e o paciente apresentou melhora expressiva da sintomatologia prévia, recebendo alta hospitalar 48 horas após o procedimento.

\section{REFERÊNCIAS}

1. Resnick D, Niwayama G. Radiographic and pathologic features of spinal involvement in diffuse idiopathic skeletal hyperostosis (DISH). Radiology. 1976;119(3):559-68.

2. Forestier J, Rotes-Querol J. Senile ankylosing hyperostosis of the spine. Ann Rheum Dis. 1950;9(4):321-30.

3. Fornasier VL, Littlejohn G, Urowitz MB, Keystone EC, Smythe HA. Spinal entheseal new bone formation: the early changes of spinal diffuse idiopathic skeletal hyperostosis. J Rheumatol. 1983;10(6):939-47.

4. Artner J, Leucht F, Cakir B, Reichel H, Lattig F. Diffuse idiopathic skeleta hyperostosis: current aspects of diagnostics and therapy. Orthopade. 2012;41(11):916-22.

5. Sencan D, Elden H, Nacitarhan V, Sencan M, Kaptanoglu E. The prevalence of diffuse idiopathic skeletal hyperostosis in patients with diabetes mellitus. Rheumatol Int. 2005;25(7):518-21.

6. Inman RD. The spondyloarthropathies. In: Goldman L, Ausiello D, editors. Cecil Textbook of Medicine. 22nd ed. Philadelphia: WB Saunders; 2004. p.1654-60.

7. Ohki M. Dysphagia due to Diffuse Idiopathic Skeletal Hyperostosis. Case Rep Otolaryngol. 2012;2012:123825. 